\title{
Dynamic Pricing of Perishable Products under Consumer Factor
}

\author{
Yanming Ge, Jianxin Zhang \\ ${ }^{1}$ College of IT, Shanghai Ocean University, Shanghai, China. \\ E-mail: \{ymge, jxzhang\}@shou.edu.cn
}

Received September $5^{\text {th }}, 2011$; revised October $16^{\text {th }}$, 2011; accepted November $10^{\text {th }}, 2011$.

\begin{abstract}
With effect of consumer factor considered, a model for dynamic pricing of perishable products is proposed. By this model, we obtained a property of the value function: marginal value is a decreasing function of the capacity and an increasing function of the consumer factor. Basing on this property, we proposed the following pricing strategy: according to the regions that the perishable products are sold, we adopt the appropriate consumer factor and then use the routine pricing strategy. This strategy not only maximizes the retailer's revenue but also improves its service level. Lastly, a constructive example is discussed.
\end{abstract}

Keywords: Revenue Management, Dynamic Pricing, Perishable Product, Bellman Equation

\section{Introduction}

Since the adoption of reform and opening-up policy, China residents' consumptive level has been dramatically increasing as a whole. In the meanwhile, owing to the difference of history and culture, the consumptive level in different regions differs greatly. Furthermore, this trend is still upwards. Although some rules and regulations were drawn up by Chinese government in order to expand domestic demand, the status quo is not changed but aggravated. Under these circumstances the retailers should take what kind of price strategies to maximize their own revenues and respond to the call of the government (in other word: improve the service level). I will discuss this problem in this paper.

There are many perishable products in reality, such as: meats, drugs, fashionable dress. Usually, perishability is classified into two categories [1]: fixed lifetime and random lifetime. In this paper we will restrict attention only to the former category.

The papers related to optimal pricing for perishable product are abundant. Gallego and van Ryzin [2] constructed a continuous-time, time-homogeneous stochastic model to analyze some properties of optimal price, give an exact solution in the exponential demand case, and prove

\footnotetext{
*This paper is supported by program of Shanghai Ocean University (No B-8515-10-0001) and Public welfare industry special funds research projects of The Ministry of Science and Technology of the P.R.C. (No. 200905014-06).
}

the asymptotic optimality of the deterministic policy. Bitran and Mondschein [3] analyzed a discrete-time pricing model and tested in on apparel retail data. Zhao and Zheng [4] discussed the continuous-time model with a time-varying demand function and provided results on the monotonicity of optimal prices over time. Das Varmand and Vettas [5] analyzed the problem of selling a finite supply over an infinite horizon with discounted revenues, where the discounting provides an incentive to sell items sooner rather than later. Feng and Gallego [6] discussed an interesting stochastic model of dynamic pricing where demand is Markovian and may depend on the current inventory level. Zhang and Cooper [7] model a pricing control problem. They acknowledge the complexity of the dynamic program, construct heuristics, and test performance using a numerical study. Dong et al. [8] examine both the initial inventory and subsequent dynamic pricing decisions with a multinomial logit model of consumer choice. Their work focuses on horizontally differentiated products, uses numerical experiments to demonstrate the value of dynamic pricing, and illustrates the value of their approach in determining near-optimal initial inventories.

The main method used in papers [2,6,7,9] is the dynamic programming. There the terminal functions of optimality equation are usually equal to zero despite how many products are left if the salvage value is not considered. We will use the same method with different terminal 
functions in this paper. In addition to obtaining the maximum revenue, our aim is to control the service level, and it is directly reflected by the number of unsold product at terminal time. Therefore the terminal function defined in this paper adopts the following strategy: if the number of unsold product is more than a given number, some "penalty" will be implemented, and the poorer the region that the stores link is, the more "penalty" is implemented. The degree of "penalty" is negatively correlated to a parameter, called consumer factor, which is a function that positively correlated to the consumption levels of the regions. Using this terminal function, the optimality equation and some properties relating to region factors are obtained: marginal value is a decreasing function of the capacity and an increasing function of the consumer factor. Basing on these properties, the strategy, which not only maximizes the retailer's revenue but also improves its service level, is given.

The remainder of the paper is organized as follows. In Section 2, we describe a dynamic pricing model. In Section 3, we investigate some properties of the expected value function. In Section 4, we present a numerical experiment to illustrate properties of the model. Concluding this study, we give our conclusions in Section 5.

\section{Proposed Model}

\subsection{Preliminaries}

Consider a firm that sells $M$ (discrete) items of a kind of perishable product in $T$ periods. Here we assume there is only one customer per period and the customer in period $t$, where $t=1,2, \cdots, T$, has a willingness to pay $v_{t}$; that is, a random variable with distribution

$$
F(t, v)=P\left(v_{t} \leq v\right)
$$

Therefore, if the firm offers a price $p$ in period $t$, it will sell exactly one unit if $v_{t}>p$ (with probability $1-F(t, p))$. Letting

$$
d(t, p)=1-F(t, p)
$$

denote the demand rate, we can define an inverse-demand function

$$
p(t, d)=F_{t}^{-1}(1-d(t))
$$

and revenue-rate function is

$$
r(t, d)=d p(t, d) \text {. }
$$

The inventory and demand in this case are both assumed to be discrete. On the other hand, let

$$
J(t, d)=\frac{\partial}{\partial d} r(t, d),
$$

and assume it is strictly decreasing in the demand $d$. Equivalently, it is strictly increasing in the price $p$.

Letting $V_{t}(n)$ denote the optimal expected revenue to go, if there is $n$ items of products unsold, and called value function.

Letting $V_{T+1}(n)$ express the expected revenue at the end of selling season (terminal time), and called terminal function, which will be defined in the following subsection.

\subsection{Definition of Terminal Function}

In traditional revenue management, if salvage cost does not consider, the expected revenue at terminal time is defined as zero usually. It is rational because the selling is stop at terminal time. However, if the region that is poor, the consumption level is very low, some products are unsold. Neither sellers nor potential customers want to see this situation.

Here we adopt a strategy to control the number of unsold product, if it is more than a given number, some "penalty" will be implemented. Firstly, let a parameter $\alpha$ for $0 \leq \alpha \leq 1$ corresponding to the region that the seller links, which is called consumer factor and positively correlated to the consumption level of this region, such as: if the regions are Shanghai or Beijing, $\alpha$ is 1 , and $\alpha$ is 0.3 if they are Xining or Guiyan (they are two cities in China). Secondly, we define a function related to the consumer factor, if the ratio of unsold product is less than the region factor, the value of this function is zero, otherwise is decreasing to the number of unsold product. This is just the terminal function we want to define. For convenience, in this paper it is defined as

$$
h(n)=-A(n-[\alpha M])^{+}
$$

where $A$ is a positive constant and $[x]$ expresses the maximum integer number less than $x$. Obviously, when $\alpha=1$, the terminal function is zero, this is just the "traditional” terminal function.

\subsection{Optimality Equation}

In order to find the optimal policy, here we give out the optimality equation of this problem, that is the following Bellman Equation:

$$
V_{t}(n)=\max _{d \geq 0}\left\{r(t, d)-d \Delta V_{t+1}(n)\right\}+V_{t+1}(n)
$$

with boundary conditions $V_{T+1}(n)=h(n)$ and $V_{t}(0)=0$ for all $t$, where $\Delta V_{t}(n)=V_{t}(n)-V_{t}(n-1)$ is the expected marginal value of capacity.

Under the monotonic assumption of $J(t, d)$, necessary and sufficient condition for the optimal rate $d^{*}$ are

$$
J(t, d)=\Delta V_{t+1}(n)
$$

Different values of $\alpha$ reflect different regional condition and thus, lead to distinct control policies. Therefore, for clarity, the consumer factor parameter will ap- 
pear as a parameter in various notations. For example, $V_{t}(\alpha, n)$ denotes the value function with the consumer factor equals to $\alpha$.

\section{Properties of the Value Function}

From Section 2, we are aware that the differences of the value function, which represent the marginal revenues of remaining capacity, play a critical role in making optimal decisions. In this section, we explore the structural properties of the value function in Theorem 1, and another property relating to consumer factor in Theorem 2 .

Theorem 1. $\Delta V_{t}(\alpha, n+1) \leq \Delta V_{t}(\alpha, n)$.

Proof: The proof is by induction on $t$.

First, when $t=T+1$, from the definition of terminal function, we have

$$
\begin{aligned}
& \Delta V_{t}(\alpha, n+1)-\Delta V_{t}(\alpha, n+1) \\
= & \left\{\begin{array}{cc}
A([\alpha M]-n-1) & n<[\alpha M] \leq n+1 \\
A(n-1-[\alpha M]) & n-1<[\alpha M] \leq n \\
0 & \text { other }
\end{array}\right.
\end{aligned}
$$

It is true obviously in this case.

Assume it is true for period $t+1$, and consider period $t$. Let $d_{i}^{*}$ denote the optimal solution to Bellman equation (1) for inventory level $n+i$. From Bellman equation we have

$$
\begin{aligned}
& \Delta V_{t}(\alpha, n+2)-\Delta V_{t}(\alpha, n+1) \\
= & \Delta V_{t+1}(\alpha, n+2)-\Delta V_{t+1}(\alpha, n+1) \\
& +r\left(t, d_{2}^{*}\right)-d_{2}^{*} \Delta V_{t+1}(\alpha, n+2) \\
& -r\left(t, d_{1}^{*}\right)-d_{1}^{*} \Delta V_{t+1}(\alpha, n+1) \\
& -r\left(t, d_{1}^{*}\right)-d_{1}^{*} \Delta V_{t+1}(\alpha, n+1) \\
& +r\left(t, d_{0}^{*}\right)-d_{0}^{*} \Delta V_{t+1}(\alpha, n)
\end{aligned}
$$

From the optimality of $d_{1}^{*}$, the following inequalities hold:

$$
\begin{array}{r}
r\left(t, d_{1}^{*}\right)-d_{1}^{*} \Delta V_{t+1}(\alpha, n+1) \\
\geq \quad r\left(t, d_{2}^{*}\right)-d_{2}^{*} \Delta V_{t+1}(\alpha, n+1)
\end{array}
$$

and

$$
\begin{array}{r}
r\left(t, d_{1}^{*}\right)-d_{1}^{*} \Delta V_{t+1}(\alpha, n+1) \\
\geq \\
r\left(t, d_{0}^{*}\right)-d_{0}^{*} \Delta V_{t+1}(\alpha, n+1)
\end{array}
$$

Substituting into (3), rearranging and canceling terms yields

$$
\begin{aligned}
& \Delta V_{t}(\alpha, n+2)-\Delta V_{t}(\alpha, n+1) \\
\leq & \left(1-d_{2}^{*}\right)\left[\Delta V_{t+1}(\alpha, n+2)-\Delta V_{t+1}(\alpha, n+1)\right] \\
& +d_{0}^{*}\left[\Delta V_{t+1}(\alpha, n+1)-\Delta V_{t+1}(\alpha, n)\right]
\end{aligned}
$$

By induction

$$
\begin{gathered}
{\left[\Delta V_{t+1}(\alpha, n+2)-\Delta V_{t+1}(\alpha, n+1)\right] \leq 0} \\
{\left[\Delta V_{t+1}(\alpha, n+1)-\Delta V_{t+1}(\alpha, n)\right] \leq 0}
\end{gathered}
$$

And since $d$ values at most one, $1-d_{2}^{*} \geq 0$ and $d_{0}^{*} \geq 0$. Therefore this conclusion is true.

This conclusion has intuitive implications for the optimal price. Note that equation (2) and Assumption $(J(t, d)$ is strictly increasing in the price $p$ ) together imply that higher marginal values correspond to higher optimal prices. Hence the more capacity remaining at any given point in time, the lower the optimal price.

Theorem 2. If $0 \leq \alpha<\beta \leq 1$, then

$$
\Delta V_{t}(\alpha, n) \leq V_{t}(\beta, n)
$$

Proof: The proof is by induction on $t$.

First, when $t=T+1$, from the definition of terminal function, if $[\alpha M]<[\beta M]-1$, we have

$$
\begin{gathered}
\Delta V_{t}(\alpha, n+1)-\Delta V_{t}(\beta, n+1) \\
\left\{\begin{array}{cc}
0 & {[\beta M] \leq n<[\alpha M]-1} \\
A(n-[\beta M]) & {[\beta M]-1 \leq n<[\beta M]} \\
-A & {[\alpha M] \leq n<[\beta M]-1} \\
A([\alpha M]-n-1) & {[\alpha M]-1 \leq n<[\alpha M]}
\end{array}\right.
\end{gathered}
$$

Therefore, it is true in this case. If $[\alpha M] \geq[\beta M]-1$, the conclusion can be proved similarly.

Assume it is true for period $t+1$ and consider period $t$. Let $d_{i \alpha}^{*}$ denote the optimal solution to Bellman equation (1) for inventory level $n+i$ and consumer factor is $\alpha$. From Bellman equation we have

$$
\begin{aligned}
& \Delta V_{t}(\alpha, n+1)-\Delta V_{t}(\beta, n+1) \\
= & \Delta V_{t+1}(\alpha, n+1)-\Delta V_{t+1}(\beta, n+1) \\
& +r\left(t, d_{1 \alpha}^{*}\right)-d_{1 \alpha}^{*} \Delta V_{t+1}(\alpha, n+1) \\
& -r\left(t, d_{0 \alpha}^{*}\right)-d_{0 \alpha}^{*} \Delta V_{t+1}(\alpha, n) \\
& -r\left(t, d_{1 \beta}^{*}\right)-d_{1 \beta}^{*} \Delta V_{t+1}(\beta, n+1) \\
& +r\left(t, d_{0 \beta}^{*}\right)-d_{0 \beta}^{*} \Delta V_{t+1}(\beta, n)
\end{aligned}
$$

From the optimality of $d_{1 \beta}^{*}$ and $d_{0 \alpha}^{*}$, the following inequalities hold:

$$
\begin{aligned}
& r\left(t, d_{1 \beta}^{*}\right)-d_{1 \beta}^{*} \Delta V_{t+1}(\beta, n+1) \\
\geq & r\left(t, d_{1 \alpha}^{*}\right)-d_{1 \alpha}^{*} \Delta V_{t+1}(\beta, n+1)
\end{aligned}
$$

and

$$
\begin{aligned}
& r\left(t, d_{0 \alpha}^{*}\right)-d_{0 \alpha}^{*} \Delta V_{t+1}(\alpha, n) \\
\geq & r\left(t, d_{0 \beta}^{*}\right)-d_{0 \beta}^{*} \Delta V_{t+1}(\beta, n)
\end{aligned}
$$

Substituting into (4), rearranging and canceling terms yields 


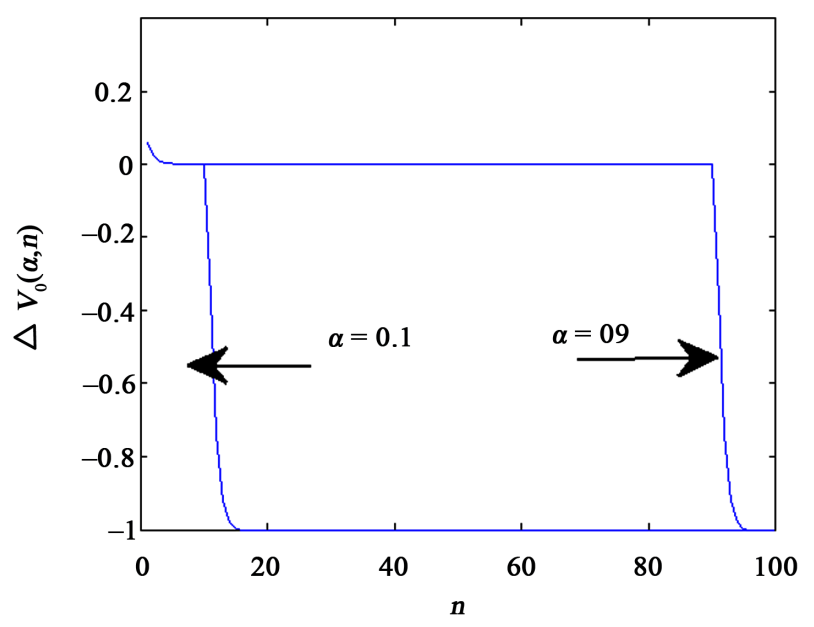

Figure 1. The curves for expected marginal value relating to consumer factor $\alpha$.

$$
\begin{aligned}
& \Delta V_{t}(\alpha, n+1)-\Delta V_{t}(\beta, n+1) \\
\leq & \left(1-d_{1 \alpha}^{*}\right)\left[\Delta V_{t+1}(\alpha, n+1)-\Delta V_{t+1}(\beta, n+1)\right] \\
+ & d_{0 \beta}^{*}\left[\Delta V_{t+1}(\alpha, n)-\Delta V_{t+1}(\beta, n)\right]
\end{aligned}
$$

By induction,

$$
\left[\Delta V_{t+1}(\alpha, n+1)-\Delta V_{t+1}(\beta, n+1)\right] \leq 0
$$

and

$$
\left[\Delta V_{t+1}(\alpha, n)-\Delta V_{t+1}(\beta, n)\right] \leq 0
$$

and since $d$ values at most one, $1-d_{1 \alpha}^{*} \geq 0$ and $d_{0 \beta}^{*} \geq 0$. Therefore, this conclusion is true.

This conclusion is just that we want to achieve. When the time and capacity is fixed, $\Delta V_{t}(\alpha, n) \leq \Delta V_{t}(\beta, n)$ means the lower the region's consumption level is, the lower price are adopted. This not only reduces the number of unsold products but also improves the service level.

\section{Numerical Results}

We present a numerical experiment in this section to show how the proposed model works in the constructive example. The results enhance the readers to understand the theoretical conclusions from the practical applications.

In this example,

Let $M=100, T=10000, d(t, p)=1.1 e^{-p}$, and $A=1$. Using the approximate numerical methods of the ordinary differential equations, we derive the following results.

Figure 1 draws curves for $\Delta V_{0}(0.1, n)$, and $\Delta V_{0}(0.9, n)$ of $n$. From these curves, it's clear that along with the increase of $n$, the marginal value is decrease when time and consumer factor are fixed, this is just the conclusion of theorem 1 . At the same time, we can observe that the curve of $\Delta V_{0}(0.9, n)$ locates over the curve of $\Delta V_{0}(0.1, n)$, this is just the conclusion of Theorem 2 .

\section{Conclusions}

This paper presents a dynamic programming model for a kind of perishable product, in which the consumer factor is considered. This model retains the desirable property in the traditional dynamic pricing models which do not consider the consumer factors: the monotone property of the marginal values. Therefore, this model does not incur any additional difficulty for its implementation.

Meanwhile, the influence of the consumer factor to the marginal values is corroborated strictly. This property guarantee that we can increase the service levels by controlling the consumer factor rationally.

Nevertheless, there are rooms to continue work on this issue. The solution procedure is not at a high satisfaction level of efficiency, especially when the capacities of the product are large. Effective heuristics are desired in response to prompt on-line enquires and transactions.

\section{Acknowledgements}

The authors thank anonymous referees and associate editor for many constructive suggestions that greatly improved the manuscript.

\section{REFERENCES}

[1] S. Nahmias, "Perishable Inventory Theory: A Review," Operations Research, Vol. 30, No. 4, 1980, pp. 680-708. doi:10.1287/opre.30.4.680

[2] G. Gallego and G. Van Ryzin, “Optimal Dynamic Pricing of Inventories with Stochastic Demand over Finite Horizons,” Management Science, Vol. 40, No. 8, 1994, pp. 999-1018. doi:10.1287/mnsc.40.8.999

[3] Britan and V. S. Mondschiin, "Periodic Pricing of Seasonal Products in Retailing," Management Science, Vol. 43, No. 1, 1997, pp. 64-79.

[4] W. Zhao and Y. S. Zheng, "Optimal Dynamic Pricing for Perishable Assets with Non-Homogeneous Demand," Management Science, Vol. 46, No. 3, 2000, pp. 375-388.

[5] G. Das Varma and N. Vettas, "Optimal Dynamic Pricing with Inventories,” Economics Letters, Vol. 72, 2001, pp. 335-340. doi:10.1016/S0165-1765(01)00439-6

[6] Y. Feng and G. Gallego, "Perishable Asset Revenue Management with Markovian Time Dependent Demand Intensities,” Management Science, Vol. 46, No. 7, 2000, pp. 941-956. doi:10.1287/mnsc.46.7.941.12035

[7] D. Zhang and W. L. Cooper, "Pricing Substitutable Flights in Airline Revenue Management," European Journal of Operations Research, Vol. 197, No. 3, 2009, pp. 848-861. doi:10.1016/j.ejor.2006.10.067

[8] L. Dong, P. Kouvelis and Z. Tian, "Dynamic pricing and inventory control of substitute products", Manufacturing Service Operations Management, Vol. 11, No. 2, 2009, 
pp. 317-339. doi:10.1287/msom.1080.0221

[9] Y. Akcay, H. P. Natarajan and S. H. Xu, “Joint Dynamic Pricing of Multiple Perishable Products Under Consumer
Choice,” Management Science, Vol. 56, No. 8, 2010, pp. 1345-1361. doi:10.1287/mnsc. 1100.1178 